\title{
EPIDEMIOLOGY OF GROUP-B STREPTOCOCCAL CARRIAGE IN PREGNANT WOMEN AND NEWBORN INFANTS
}

\author{
Patricia Ferrieri, P. P. Cleary and A. E. Seeds \\ Departments of Pediatrics, Microbiology and Obstetrics, University of Minnesota Medical \\ School, Minneapolis, Minnesota 55455, USA
}

THE group-B streptococcus, or Streptococcus agalactiae, is a well-recognised pathogen in veterinary medicine because of its role as one causative agent of bovine mastitis. In more recent years it has become apparent that this organism contributes to such serious diseases as sepsis and meningitis in the newborn infant as well as to intrapartum chorioamnionitis, septic abortions, and other diverse types of human infection (Hood, Janney and Dameron, 1961; Eickhoff et al., 1964; Jelinkova, Neubauer and Duben, 1970; Eickhoff, 1972; Wilkinson Facklam and Wortham, 1973).

Although neonatal infections due to gram-negative enteric organisms continue to predominate in some areas, serious infections due to group-B streptococci compete for first place in many regions of the United States (Yow, 1974). Group-B streptococcal infections are the most common life-threatening infections due to gram-positive organisms in the newborn infant, and in some reports the group-B streptococcus has been the most frequent cause of purulent meningitis in infants aged less than 2 months (Baker et al., 1973; Barton, Feigin and Lins, 1973). It has been suggested that serious infections due to this organism have increased in frequency in recent years but the evidence for this is inadequate (Yow, 1974).

Studies in the United States and other countries support the association of genital-tract carriage of group-B streptococci in pregnant women with subsequent asymptomatic acquisition of the organisms or clinical infection in their newborn infants within days of birth (Butter and de Moor, 1967; Bergqvist et al., 1971; Harper, 1971; Baker and Barrett, 1973; Franciosi, Knostman and Zimmerman, 1973; Anthony, Okada and Hobel, 1975).

The purposes of the present investigation included (1) ascertaining the prevalence of cervico-vaginal carriage of group-B streptococci in pregnant women during the 3 rd trimester of pregnancy and at the time of labour and the relation of this to acquisition of the organisms by their newborn infants; (2) comparing the value of cervical and vaginal cultures during the 3rd trimester of pregnancy for the detection of carriers; (3) determing the frequency of conversion from a culture-negative to a culture-positive state, and vice versa, by the time of presentation in labour, and of the persistence of carriage; (4)

Received 3 June 1976; revised version accepted 23 Aug. 1976.

J. MED. MICROBIOL.-VOL. 10 (1977) 
estimating the frequency of neonatal acquisition of group-B streptococci at various body sites and the numbers of these bacteria present; and (5) studying the distribution of serotypes among the group-B streptococcal isolates from the women and their infants.

\section{MATERIALS AND METHODS}

Patients examined. Pregnant women enrolled in the Obstetric Clinic at the University of Minnesota Hospital were included in the study. All deliveries were in the same hospital with an average length of stay of 5 days for the mothers and newborn infants. The study period was from 1 Apr. 1974 to 15 Aug. 1975. All infants were assigned to one of two nurseries according to a cohort system. Special emphasis is placed in this hospital on hygienic techniques in the nursing care of the infants.

Methods of culture and bacterial processing. At about the 36th week of pregnancy, maternal endocervical and vaginal cultures were obtained; vaginal cultures only were taken on admission to the labour and delivery area. Within $3 \mathrm{~h}$ of birth, the newborn infants had cultures of the anterior nares, external ear canals, and base of the umbilical cord. Additional cultures, including gastric-aspirate fluid and blood, were obtained as indicated by the clinical condition. Blood cultures were taken frequently from infants found to be contaminated with group-B streptococci after birth. At the time of discharge from the nursery, infants had cultures of the anterior nares and umbilicus.

Cultures were taken with a rayon-tipped swab ("Culturette", Marion Scientific Corporation, Rockford, Illinois, with modified Stuart's transport medium) and were inoculated on to $6 \%$ sheep-blood-agar plates containing colistin, $10 \mu \mathrm{g}$ per $\mathrm{ml}$, and nalidixic acid, $15 \mu \mathrm{g}$ per $\mathrm{ml}$ (Columbia CNA Agar, Difco Laboratories, USA). After incubation for c. $18 \mathrm{~h}$ aerobically at $35^{\circ} \mathrm{C}$, the plates were examined and the number of colonies of $\beta$ haemolytic streptococci recorded. Negative plates were re-examined at $48 \mathrm{~h}$. Two colonies of $\beta$-haemolytic streptococci, when present, were subcultured for further identification. Streptococci that were not $\beta$-haemolytic were also occasionally selected for further identification. All streptococci were grouped serologically (Lancefield, 1933). All group-B streptococcal isolates were typed by the capillary precipitin method (Lancefield, 1934; Swift, Wilson and Lancefield, 1943; Wilkinson et al., 1973) or by an adaptation of the

TABLE I

Prevalence of group-B streptococci among pregnant women and newborn infants

\begin{tabular}{|c|c|c|}
\hline Category of person & $\begin{array}{l}\text { Number of } \\
\text { persons } \\
\text { examined }\end{array}$ & $\begin{array}{c}\text { Number (and percentage) } \\
\text { of persons giving a } \\
\text { positive culture* }\end{array}$ \\
\hline $\begin{array}{l}\text { Women; 3rd trimester } \uparrow \\
\text { Women; in labour } \ddagger \\
\text { Infants; at birth§ } \\
\text { Infants; negative at birth, } \\
\text { swabbed on discharge }\end{array}$ & $\begin{array}{l}802 \\
759 \\
900 \\
869\end{array}$ & $\begin{array}{l}45(5 \cdot 6) \\
63(8 \cdot 3) \\
31(3 \cdot 4) \\
19(2 \cdot 2)\end{array}$ \\
\hline
\end{tabular}

* The women or infants were counted as positive if any of the indicated cultures (see below) were positive.

$\dagger$ Vagina and cervix $=796$; vagina only $=5$, cervix only $=1$.

$\ddagger$ Vagina.

$\S$ Nares, ears and umbilicus.

If Nares and umbilicus. 
double-diffusion method in agar gel (Rotta et al., 1971). Grouping and typing antisera were supplied by the Center for Disease Control, Atlanta, Georgia.

\section{RESULTS}

In table I, the prevalence rates for group-B streptococcal carriage in pregnant women and their infants are given. A relatively low genital carriage rate $(5.6 \%)$ was seen during the 3rd trimester of pregnancy. This had increased to $8.3 \%$ at the time of culturing in labour, mainly because of the conversion to positive of 20 persons who had given negative cultures during the 3rd trimester. At birth, $3.4 \%$ of newborn infants had one or more surface cultures positive for group-B streptococci. An additional $2.2 \%$ of the infants who had given negative swabs at birth were culture positive at the time of discharge from the hospital. The 31 infants who gave positive swabs at birth were cultured at discharge and 14 of them gave negative swabs.

\section{Pattern of carriage of group-B streptococci by pregnant women}

Carriage in the $3 r d$ trimester of pregnancy. Both cervical and vaginal swabs were obtained from 796 women in the 3 rd trimester. Of 41 culture-positive women, $25(61 \%)$ yielded group-B streptococci from both sites, $11(27 \%)$ from the vagina only, and five (12\%) from the cervix only.

Persistence or change of carriage status in parturients. Of 63 women who gave a positive vaginal swab when examined in labour at term, the previous carriage status was known in 48, and of these $20(42 \%)$ had given negative swabs in the 3 rd trimester. Of 45 women who had given a positive vaginal or

\section{TABLE II}

Neonatal acquisition of group-B streptococci in relation to carriage status of the mother

\begin{tabular}{|c|c|c|}
\hline \multirow{2}{*}{ Carriage status of mother } & \multicolumn{2}{|c|}{$\begin{array}{c}\text { Frequency of acquisition* by } \\
\text { infants }\end{array}$} \\
\hline & at birth & $\begin{array}{l}\text { at birth or on } \\
\text { discharge, or both }\end{array}$ \\
\hline $\begin{array}{l}\text { Third trimester: } \\
\text { vagina }+; \text { cervix }+ \\
\text { vagina }+; \text { cervix }- \\
\text { vagina }-; \text { cervix }+ \\
\text { In labour: } \\
\text { vagina }+ \\
\text { vagina }- \text { (all) } \\
\text { vagina }- \text { (but }+ \text { in 3rd } \\
\text { trimester } \dagger \text { ) }\end{array}$ & $\begin{array}{c}10 / 25(40) \\
2 / 11(18) \\
1 / 5 \quad(20) \\
23 / 63(37) \\
3 / 689(0 \cdot 4) \\
0 / 7 \quad(0)\end{array}$ & $\begin{array}{c}13 / 25(52) \\
5 / 11(45) \\
1 / 5 \quad(20) \\
31 / 63(49) \\
7 / 689(1) \\
2 / 7 \quad(29)\end{array}$ \\
\hline
\end{tabular}

$+=$ Group-B streptococci isolated; $-=$ group-B streptococci not isolated.

* Number of infants yielding positive swabs/number examined (percentage).

$\uparrow$ Vagina or cervix or both. 
TABLE III

Sites from which group-B streptococci were isolated at birth

\begin{tabular}{|c|c|c|c|c|}
\hline \multirow{2}{*}{$\begin{array}{l}\text { Number of sites } \\
\text { positive }\end{array}$} & \multicolumn{4}{|c|}{$\begin{array}{l}\text { Number of infants yielding an isolation } \\
\text { from }\end{array}$} \\
\hline & ears & nose & umbilicus & $\begin{array}{l}\text { any of } \\
\text { these }\end{array}$ \\
\hline $\begin{array}{l}1 \\
2 \\
3\end{array}$ & $\begin{array}{r}8 \\
8 \\
13\end{array}$ & $\begin{array}{r}1 \\
6 \\
13\end{array}$ & $\begin{array}{r}1 \\
2 \\
13\end{array}$ & $\begin{array}{c}10^{*} \\
8^{*} \\
13 \dagger\end{array}$ \\
\hline
\end{tabular}

* Isolation from gastric aspirate also in one infant in each of these groups.

$\dagger$ Isolation from gastric aspirate also in two infants.

cervical culture (or both) in the 3rd trimester, 37 had swabs taken in labour; seven $(19 \%)$ gave a negative vaginal culture in labour.

\section{Acquisition of group-B streptococci by newborn infants}

Frequency of acquisition in relation to the carriage status of the mother. In infants born to mothers with positive cultures in the 3rd trimester, the frequency of acquisition at birth was highest $(40 \%)$ if both vagina and cervix were positive (table II). By the time of discharge from hospital, additional infant carriers were detected in all categories. A comparison of the total frequencies of acquisition of group-B streptococci (at birth or on discharge or both) reveals no significant difference between infants born to mothers with both cultures positive (13 of $25 ; 52 \%$ ) and with only a positive vaginal swab (five of 11 ; $45 \%$ ). Infants born to mothers with a positive vaginal swab in labour had a

\section{TABLE IV}

Distribution of group-B streptococcal isolates from newborn infants by site and time of culture

\begin{tabular}{l|cc}
\hline \multicolumn{1}{c|}{ Site } & $\overbrace{}^{\text {at birth }}$ & $\begin{array}{c}\text { Number (and percentage) of } \\
\text { isolates from the stated site }\end{array}$ \\
\hline $\begin{array}{c}\text { on discharge } \\
\text { from hospital* }\end{array}$ \\
$\begin{array}{l}\text { Nose } \\
\text { Umbilicus }\end{array}$ & $\begin{array}{l}20(31) \\
29(45) \\
16(25)\end{array}$ & $\begin{array}{l}12(55) \\
\text { N.D. } \\
10(45)\end{array}$ \\
\hline
\end{tabular}

* But negative cultures at birth.

$\uparrow$ Not done. 
TABLE V

Numbers of group-B streptococcal colonies isolated from various culture sites of infants at birth

\begin{tabular}{|c|c|c|c|c|}
\hline \multirow{2}{*}{ Site } & \multirow{2}{*}{$\begin{array}{l}\text { Number } \\
\text { of } \\
\text { positive } \\
\text { cultures }\end{array}$} & \multicolumn{3}{|c|}{$\begin{array}{l}\text { Percentage of positive cultures } \\
\text { from the stated site that yielded }\end{array}$} \\
\hline & & $\begin{array}{c}1-9 \\
\text { colonies }\end{array}$ & $\begin{array}{c}10-49 \\
\text { colonies }\end{array}$ & $\begin{array}{l}>50 \\
\text { colonies }\end{array}$ \\
\hline $\begin{array}{l}\text { Nose } \\
\text { Ears } \\
\text { Umbilicus }\end{array}$ & $\begin{array}{l}20 \\
29 \\
16\end{array}$ & $\begin{array}{l}35 \\
21 \\
37 \cdot 5\end{array}$ & $\begin{array}{l}20 \\
17 \\
12 \cdot 5\end{array}$ & $\begin{array}{l}45 \\
62 \\
50\end{array}$ \\
\hline
\end{tabular}

high frequency $(37 \%)$ of acquisition of streptococci at birth which increased to $49 \%$ by discharge from hospital. Of interest were the two culture-positive infants born to mothers with a negative swab in labour but positive culture in the 3rd trimester. Of 689 infants born to culture-negative mothers, only $1 \%$ acquired streptococci.

Sites of isolation of group-B streptococci. Table III presents the frequency with which group-B streptococci were isolated from ears, nose and umbilicus. In 10 of 31 infants, only one of these three sites yielded a positive culture, but positive cultures from three sites was the commonest finding. Of interest was the frequency with which external ear-canal cultures were positive. Eight of 10 infants positive at only one site had positive ear-canal cultures. All of the remaining 21 infants, positive at two or more sites, also had positive earcanal cultures.

The total number of group-B streptococcal isolates from infants at birth and at discharge from hospital and their site distribution are presented in table IV. Again, the value of culturing the external ear canal at birth is apparent$45 \%$ of all isolations at the time were made from this site. Detection of additional culture-positive infants at the time of discharge revealed a nearly equal distribution of isolations from nasal and umbilical cultures.

Numbers of group-B streptococci isolated from infants at birth. The degree of group-B streptococcal contamination of infants shortly after birth has not been previously reported. In this study, the number of colonies of group-B streptococci on each positive culture-plate was recorded semi-quantitatively. Although the percentage distribution of light, moderate and heavy contamination at the various sites was rather similar (table V), a high percentage (45-62\%) of positive cultures from all sites yielded 50 or more colonies per plate. These high colony counts suggest that the infants had been heavily contaminated with streptococci. In children with lower colony counts in nasal cultures at the time of birth, i.e., less than 10 colonies per plate, it was common to see 50 or more colonies in nasal cultures taken on discharge from hospital. 

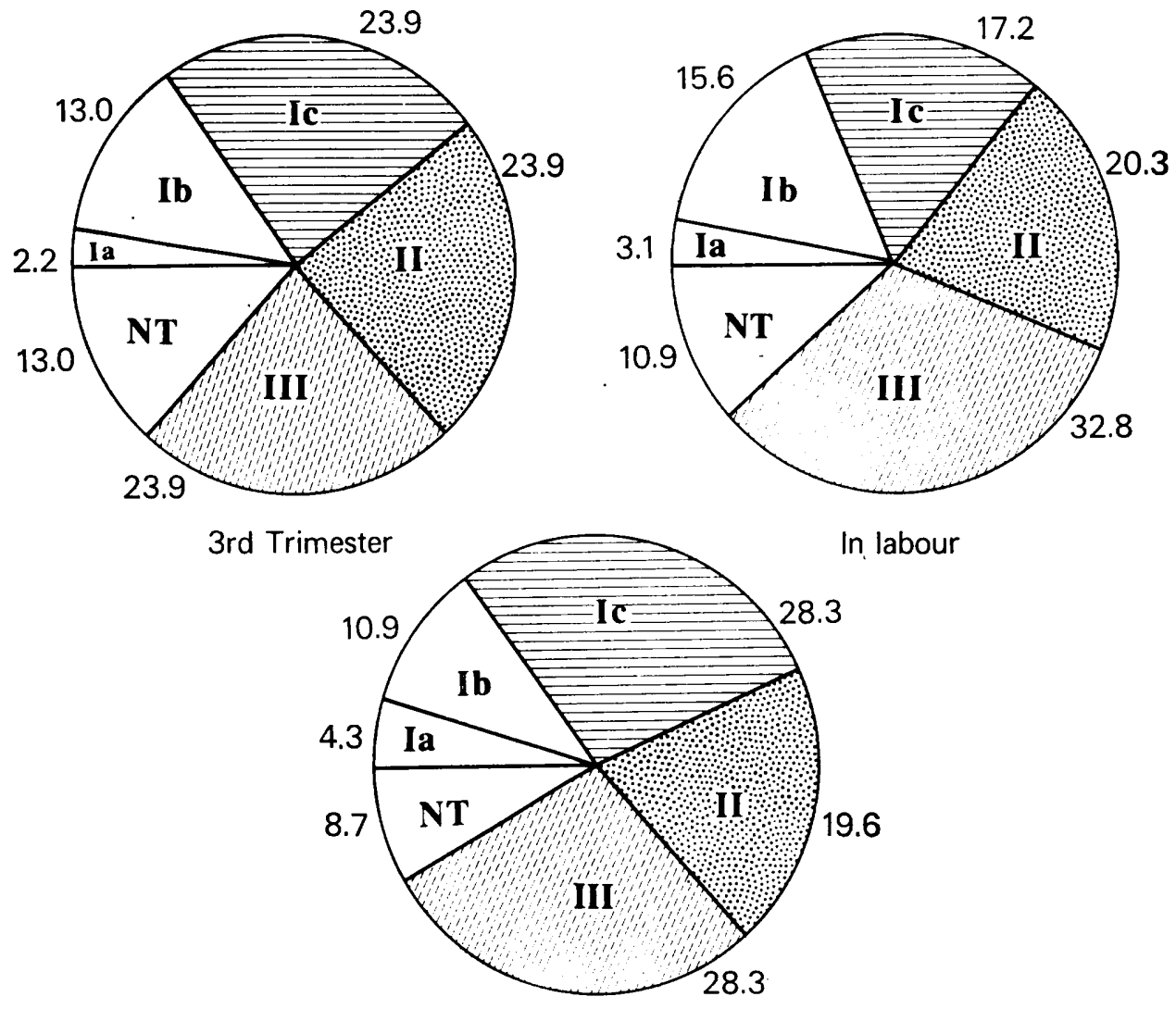

Newborn infants

FIGURE.-Type distribution among group-B streptococci from (1) women in the 3rd trimester of pregnancy, (2) women in labour and (3) infants newly born to these women: percentage of culture-positive persons who yielded streptococci of the stated types.

\section{Distribution of streptococcal serotypes}

The distribution of serotypes among group-B streptococci isolated from women, during the 3 rd trimester of pregnancy and at the time of labour, and from newborn infants, is depicted in the figure. During the 3rd trimester, group-B types Ic, II, and III were equally common and together were isolated from $72 \%$ of culture-positive persons. A rather similar distribution was observed in labour, but type-III strains were rather more common and were isolated from nearly one-third of the women. The serotype distribution of strains isolated from newborn infants paralleled the trends in the mothers, but type Ic strains were a little more common in the infants than in the mothers. The least frequently isolated serotype in both infants and mothers was type Ia.

When positive swabs were obtained in the $3 \mathrm{rd}$ trimester and at the time of labour, they usually belonged to the same serotype; this suggested persistent 
carriage of the same strain. On only one occasion were members of two serotypes isolated from the same woman. The streptococci isolated from mothers were, with one exception, identical with those subsequently isolated from their respective infants; a member of type $\mathrm{Ib}$ was isolated from the genital tract of one woman during pregnancy and at time of labour, but culture of the umbilicus of her infant on the 5th day of life yielded a type-II strain.

\section{Neonatal infections}

Blood cultures were obtained from 19 of 31 infants who had given positive surface cultures at birth. Three infants had positive blood cultures; the group-B streptococci isolated were two of type III and one of type Ic. The three infants were treated with antibiotics and showed no definite evidence of disease attributable to group-B streptococci. No other neonatal infections were discovered.

\section{Discussion}

These longitudinal epidemiological studies reveal that, in our population, a relatively low percentage $(5.6 \%)$ of women in the 3 rd trimester of pregnancy carry group-B streptococci in the genital tract. Other carriage rates in the literature, based ordinarily on cultures taken immediately before delivery, are in the range $5-12 \%$, but figures as high as $29 \%$ have been reported (Baker and Barrett, 1973; Franciosi et al., 1973; Anthony et al., 1975). Enrichment culture in broth media containing inhibitory antibiotics has been reported to increase the frequency of positive cultures; the use of this technique may account for the reported carriage rates in excess of $20 \%$ (Baker and Barrett, 1973). In the present study, in which swabs were inoculated directly on to primary plates containing colistin and nalidixic acid, other bacteria were infrequently found and when present formed only a few colonies. Recently, however, we performed a trial of enrichment in Todd-Hewitt broth containing gentamicin $(8 \mu \mathrm{g}$ per $\mathrm{ml})$ and nalidixic acid $(15 \mu \mathrm{g}$ per $\mathrm{ml})$ in which the swab was immersed in the broth and remained in it during incubation, and compared the results with those obtained by parallel cultures on inhibitory agar plates. During a 6-week period, $6 \%$ of the enrichment cultures were positive on samples that gave negative results on direct culture. Even if our carriage rates had been doubled by the use of enrichment culture, they would have been modest in comparison with some of the rates reported in the literature. The increased frequency of positive cultures obtained by enrichment in broth is undoubtedly due to the detection of small numbers of organisms embedded in the swab; the clinical significance of this is unclear. Differences are to be expected in the prevalence of streptococcal carriage in various population groups. The present population is notable in that gonococcal carriage or infection is very uncommon.

Little information is available in the literature on either the persistence of genital-tract carriage of group-B streptococci or the frequency of conversion 
of culture status. By examining parturients during the 3rd trimester of pregnancy as well as at the time of labour, it was possible to show that carriage was sustained in $81 \%$ of the women who had given a positive swab on the first occasion. Unfortunately, cultures were not obtained early in pregnancy or after delivery to follow the dynamics of acquisition and the length of persistence of the organisms over a longer period of time. The studies of Anthony et al. (1975) which included cultures taken on several occasions during pregnancy and after delivery indicated that persistent carriage was present in only $38 \%$ of his patients.

In the present study, $19 \%$ of women positive during the latter part of pregnancy were culture negative at the time of presentation in labour. Conversely, $42 \%$ of women positive in labour had given negative cultures in the 3 rd trimester. This may be attributable to the frequent acquisition of group-B streptococci in late pregnancy; alternatively, carriage may be intermittent and transient, or the organisms may not have been isolated at the first swabbing because of sampling errors or technical difficulties. Therefore, identification of the " at risk" mother-infant pair before labour may be hampered by the frequency of " conversion" of the maternal streptococcal carriage status.

There is a modest advantage to be gained by culture of both cervix and vagina during the 3 rd trimester of pregnancy. Had only a vaginal culture been taken, $88 \%$ of the culture-positive women would have been detected. Others have suggested that urethral cultures (Anthony et al., 1975) and rectal cultures (Badri et al., 1976) are more frequently positive than genital-tract cultures in pregnant women, but that throat cultures are less frequently positive (Baker and Barrett, 1973). Further studies are necessary to substantiate these findings, but the possibility that a reservoir for group-B streptococci exists in the gastrointestinal tract is interesting, and may account for the difficulty of eradicating group-B streptococcal carriage in women or their infants (Paredes et al., 1976).

Of interest in our study was the lack of a difference in the frequency of streptococcal acquisition by infants born to mothers with a positive cervical and vaginal culture and by those born to women with only a positive vaginal culture. Nearly $50 \%$ of the infants born to culture-positive mothers in labour acquired group-B streptococci either at birth or by the time of discharge from hospital. This figure is somewhat lower than the percentages $(c .60-70)$ obtained in other studies (Baker and Barrett, 1973; Anthony et al., 1975). Support for the view that acquisition by newborn infants is a consequence of contamination of the amniotic fluid with group-B streptococci, and thus occurs in utero is provided by the fact that the organism was isolated from the ear canals of 29 of 31 infants who gave a positive culture at birth. The external ear canal, relatively inaccessible to simple contamination from passage through the birth canal, probably sequesters amniotic fluid. Maternal genital-tract streptococci undoubtedly gain access to the amniotic sac by the ascending route, and the probability of this increases with the duration of labour and the length of time between rupture of the membranes and delivery. However, streptococci presumably may enter the amniotic sac in the presence of " intact " membranes, 
because newborn infants born by caesarian section may develop streptococcal sepsis within a few hours of birth (P. Ferrieri, unpublished).

Contamination of the newborn infant at multiple orifices and skin sites (nares, ear canal, umbilicus) was seen frequently; indeed, $42 \%$ of the infants from whom group-B streptococci were isolated at birth had positive cultures from at least three sites. The high proportion of positive cultures at birth that yielded 50 or more colonies of streptococci per primary culture plate suggests that bacterial contamination was heavy.

The isolation of streptococci at the time of discharge from hospital from 19 infants who had been culture negative at birth is compatible also with acquisition via the maternal genital-tract. The cultural technique employed may have been inadequate for the detection of small numbers of streptococci present at birth in minimally contaminated infants, but postnatal transmission from mother to infant cannot be ruled out.

The term "colonisation" is used frequently in referring to mothers or infants with positive cultures for group-B streptococci. We prefer the terms "carriage" or "contamination" unless there is evidence that multiplication of organisms has occurred. Some data from this study suggest that at birth the infants may be contaminated with streptococci but later become truly colonised. Nasal or umbilical cultures, for example, may yield fewer than 10 colonies per culture plate at birth but 50 or more colonies 4-5 days later, indicating actual multiplication of the organisms.

Group-B streptococci of serotypes Ic, II, and III were the most prevalent among both the mothers and their infants in our population. Elsewhere in the United States types II and III were most commonly isolated from similar persons (Baker and Barrett, 1974; Anthony et al., 1975). However, in the Netherlands, Butter and de Moor (1967) identified type Ia as predominant in infants and adults harbouring or infected with group-B streptococci. It might be expected that the distribution of group-B serotypes would vary in different geographical areas and even fluctuate within population groups over periods of time.

The close correspondence of serotype distribution in pregnant women and their infants in our study supports the concept that transmission is from mother to infant. The explanation for the one exception is unclear, but maternal carriage of two serotypes, one of which was unidentified, is a possibility. Alternatively, nosocomial acquisition of the discordant type by the infant may have occurred. The chance of neonatal acquisition was unrelated to the serotype of the strain carried by the mother.

Two types of clinical infection with group-B streptococci have been described in infants (Baker et al., 1973; Barton et al., 1973; Franciosi et al., 1973). The acute-onset or "early" type in infants aged 10 days or less is often associated with obstetric problems, particularly prolonged rupture of membranes and prematurity. There is evidence that the streptococci were acquired from the mother before or at the time of delivery. Since infants with " early"-onset infection frequently give evidence of respiratory distress and pneumonia, the portal of entry of the bacteria may be the respiratory tract. 
The lung may be a primary focus of infection with resulting bacteriaemia and dissemination of bacteria to other foci such as the meninges (Wannamaker and Ferrieri, 1975).

The delayed or "late"-onset form of infection has been described in infants 10 days to several weeks of age, and usually includes meningitis. The mode of transmission of streptococci to these infants is less clear. Maternal genital-tract transmission is thought to be unlikely, but nosocomial sources have not been excluded.

Data suggest that neonatal acquisition of group-B streptococci at birth is relatively common. The risk of serious infection at birth has been calculated to be one per 100 " colonised" infants, with total infection rate of 2-3 per 1000 live births and a mortality rate of one per 1000 live births (Baker and Barrett, 1973; Franciosi et al., 1973). In the present study we observed a zero rate for serious clinical disease and mortality due to group-B streptococci, but we surveyed less than 1000 live births and only 50 contaminated or carrier infants. However, we did observe three apparent instances of symptomless bacteriaemia among infants who had given positive surface swabs. The significance of this is uncertain, because contamination of the blood at the time of collection cannot be absolutely excluded.

\section{SUMMARY}

In a population of pregnant women, the prevalence of group-B streptococcal carriage was relatively low. During the 3rd trimester of pregnancy $5.6 \%$ of women haboured group-B streptococci and $8.3 \%$ were positive at the onset of labour. Some $42 \%$ of women who gave positive cultures in labour had given negative cultures during the $3 \mathrm{rd}$ trimester and $19 \%$ of women who were positive during late pregnancy were culture-negative in labour. The conversion of culture status observed in these women suggests that carriage may be intermittent or that new acquisition of genital-tract streptococci may occur in late pregnancy. The unpredictability of conversion diminishes the reliability of a single culture taken during the 3rd trimester of pregnancy.

Acquisition of streptococci was seen in nearly $50 \%$ of infants born to women who were culture positive in labour. The maternal site of carriage (cervix or vagina or both) did not appear to influence the likelihood of bacterial acquisition by the infants. The isolation of streptococci from multiple sites was quite common in the infants, and the intensity of bacterial contamination was usually high. The external ear canal was a favourable site for detecting neonatal contamination with group-B streptococci; $94 \%$ of infants, who gave positive cultures at birth had positive ear-canal cultures, and in $26 \%$ the ear canal was the only site from which streptococci were isolated. This suggests that bacterial contamination of amniotic fluid by the ascending route was a common event in culture-positive women. Three infants apparently had asymptomatic streptococcal bacteriaemia.

Isolation of the same serotype of group-B streptococcus from the infants and their mothers, and the presence of the organism in the infants-often in 
large numbers-shortly after birth, support the view that the organism is usually transmitted from the maternal genital tract to the infant.

There was no evidence that any one type was more likely than others to be transmitted from mother to infant. Group-B streptococci of serotypes Ic, II, and III were the predominant ones in the infants and their mothers in this study.

We thank the participants in the study and the medical and nursing staff of the Obstetrics Department, University of Minnesota, for their cooperation. We also thank Joanne Kumagai, JoAnn Nelson, Margaret Ragan, and D. Johnson for their invaluable assistance.

We express our gratitude to Dr Lewis W. Wannamaker for his encouragement and interest and for his critical review of the manuscript.

This work was supported by a research grant from the US Public Health Service (AI09527). P. F. was supported in part by a grant from the US Public Health Service (HL06314-15) during the period of this study.

\section{REFERENCES}

ANThony, B. F., OKaDA, D. AND HoBel, C. J. 1975. Group B streptococci (GBS) in perinatal infections: natural history of maternal and neonatal colonization. Ped. Res., 9, 296 (abstract).

Badri, M. S., Zawaneh, S., Cruz, A., Baer, H., Spellacy, W. N. and Ayoub, E. M. 1976. Rectal colonization with group B streptococci. Ped. Res., 10, 394 (abstract).

BAKER, C. J. AND BARRETT, F. F. 1973. Transmission of group B streptococci among parturient women and their neonates. J. Pediat., 83, 919.

BaKer, C. J. AND Barret, F. F. 1974. Group B streptococcal infections in infants: the importance of the various serotypes. J. Am. med. Ass., 230, 1158.

Baker, C. J., BarRett, F. F., Gordon, R. C. AND Yow, M. D. 1973. Suppurative meningitis due to streptococci of Lancefield group B: a study of 33 infants. J. Pediat., 82, 724.

Barton, L. L., Feigin, R. D. AND Lins, R. 1973. Group B beta hemolytic streptococcal meningitis in infants. J. Pediat., 82, 719.

Bergqvist, G., Hurvell, B., Thal, E. and Vaclavinkova, V. 1971. Neonatal infections caused by group B streptococci. Relation between the occurrence in the vaginal flora of term pregnant women and infection in the newborn infant. Scand. J. infect. Dis., 3, 209.

ButTER, M. N. W. AND DE Moor, C. E. 1967. Streptococcus agalactiae as a cause of meningitis in the newborn, and of bacteraemia in adults. Differentiation of human and animal varieties. Antonie van Leeuwenhoek, 33, 439.

EICKHOFF, T. C. 1972. Group B streptococci in human infection. In Streptococci and streptococcal diseases, recognition, understanding, and management, edited by L. W. Wannamaker and J. M. Matsen, New York, p. 533.

Eickhoff, T. C., Klein, J. O., Daly, A. K., Ingall, D. and Finland, M. 1964. Neonatal sepsis and other infections due to group B beta-hemolytic streptococci. N. Engl. J. Med., 271, 1221.

Franciosi, R. A., Knostman, J. D. And Zimmerman, R. A. 1973. Group B streptococcal neonatal and infant infections. J. Pediat., 82, 707.

HARPER, I. A. 1971. The importance of group B streptococci as human pathogens in the British Isles. J. clin. Path., 24, 438.

HOOD, M., JANNEY, A. AND DAMERON, G. 1961. Beta hemolytic streptococcus group B associated with problems of the perinatal period. Am.J. Obstet. Gynec., 82, 809.

Jelinkova, J., Neubauer, M. AND Duben, J. 1970. Group B streptococci in human pathology. Zentbl. Bakt. ParasitKde, I Abt. Orig., 214, 450.

LANCEFIELD, R. C. 1933. A serological differentiation of human and other groups of hemolytic streptococci. J. exp. Med., 57, 571.

LANCEFIELD, R. C. 1934. A serological differentiation of specific types of bovine hemolytic streptococci (group B). J. exp. Med., 59, 441. 
Paredes, A., Wong, P. And Yow, M. D. 1976. Failure of penicillin to eradicate the carrier state of group B streptococcus in infants. J. Pediat., 89, 191.

Rotta, J., Krause, R. M., Lancefield, R. C., Everly, W. ANd Lackland, H. 1971. New approaches for the laboratory recognition of $\mathbf{M}$ types of group A streptococci. J. exp. Med., 134, 1298.

Swift, H. F., Wilson, A. T. AND LanCefield, R. C. 1943. Typing group A hemolytic streptococci by $\mathrm{M}$ precipitin reactions in capillary pipettes. J. exp. Med., 78, 127.

Wannamaker, L. W. AND Ferrieri, P. 1975. Streptococcal infections-updated. DiseaseA-Month, October, Chicago, p. 26.

Wilkinson, H. W., FACKLAM, R. R. AND Wortham, E. C. 1973. Distribution by serological type of group B streptococci isolated from a variety of clinical material over a five-year period (with special reference to neonatal sepsis and meningitis). Infect. Immun., 8, 228.

Yow, M. 1974. Group B streptococci: a serious threat to the neonate. J. Am. med. Ass., $230,1177$. 